18. Schlömilch, développement de quelques intégrales définies.

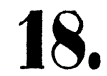

\title{
Développement de quelques intégrales définies, renfermant des fonctions trigonométriques.
}

(Par Mr. le Dr. O. Schlömilch, professeur à l'université de Jena.)

Depuis longtemps les deux formules $_{\text {en }}$

$$
\begin{aligned}
& \text { 1. } \int_{0}^{\infty} x^{\mu-1} e^{-x} \cos u x \partial x=\frac{\Gamma(u) \cos (\mu \operatorname{arctang} u)}{\left(1+u^{2}\right)^{\frac{1}{2} u}}, \\
& \text { 2. } \int_{0}^{\infty} x^{\mu-1} e^{-x} \sin u x \partial x=\frac{\Gamma(u) \sin (u \operatorname{arctang} u)}{\left(1+u^{2}\right)^{\frac{1}{2} u}}
\end{aligned}
$$

sont connues, mais on n'a pas encore essayé d'en tirer des nouvcaux résultats pour la théorie des intégrales définies. Or ces formules donnent différentes intégrales définies, si on les combine avec d'autres formules du même genre. Nous présenterons ici quelques exemples de ces combinaisons.

I.

Après avoir remplacé $\mu$ par $p$, nous multiplierons l'équation (1.) par $\frac{\partial u}{u^{q}}$ et en prendrons lintégrale par rapport à $u$, entre les limites $u=0$ et $u=\infty$. Cela donue

3. $\int_{0}^{\infty} \frac{\partial u}{u^{q}} \int_{0}^{\infty} x^{p-1} e^{-x} \cos u x \partial x=\Gamma(p) \int_{0}^{\infty} \frac{\cos (p \operatorname{arctang} u)}{\left(1+u^{2}\right)^{\frac{1}{2} p}} \cdot \frac{\partial u}{u^{q}}$.

Quant à l'intégrale double à gauche, on en peut trouver la valeur en renversant l'ordre des intégrations. Par cela elle se change en

$$
\int_{0}^{\infty} x^{p-1} e^{-x} \partial x \int_{0}^{\infty} \frac{\cos x u}{u^{q}} \partial u
$$

et en posant $u=\frac{t}{x}$, l'intégrale double se change en un produit de deux intégrales simples, savoir en

$$
\left(\int_{0}^{\infty} x^{p+q-2} e^{-x} \partial x\right)\left(\int_{0}^{\infty} \frac{\cos t}{t^{q}} \partial t\right),
$$

c’est à dire en

$$
\Gamma(p+q-1) \cdot \frac{\pi}{2 \Gamma(q) \cos \frac{1}{2} q \pi}, \quad 1>q>0 .
$$

Crelle's Journal f. d. M. Bd. XXXIII. Heft 4. 
Car comme $\frac{1}{t^{q}}=\frac{1}{\Gamma(q)} \int_{0}^{\infty} z^{q-1} e^{-t_{z}} \partial z$, il suit delà

$$
\int_{0}^{\infty} \frac{\cos t}{t^{q}} \partial t=\frac{1}{\Gamma(q)} \int_{0}^{\infty} \cos t \partial t \int_{0}^{\infty} z^{q-1} e^{-t z} \partial z
$$

et en renversant l'ordre des intégrations:

$$
\int_{0}^{\infty} \frac{\cos t}{t^{4}} \partial t=\frac{1}{\Gamma(q)} \int_{0}^{\infty} z^{q-1} \partial z \int_{0}^{\infty} e^{-z t} \cos t \partial t=\frac{1}{\Gamma(q)} \int_{0}^{\infty} \frac{z^{q} \partial z}{1+z^{2}} .
$$

La valeur de l'intégrale à droite se trouve à l'aide de la formule connue

donc on ubtient

$$
\int_{0}^{\infty} \frac{z^{n-1} \partial z}{1+z^{n}}=\frac{\pi}{n \sin \frac{m \pi}{n}}, \quad n>m>1,
$$

$$
\int_{0}^{\infty} \frac{\cos t}{t^{4}} \partial t=\frac{1}{\Gamma(q)} \cdot \frac{\pi}{2 \sin \frac{1}{2}(q+1) \pi}=\frac{\pi}{2 \Gamma(q) \cos \frac{1}{2} q \pi}, \quad 1>q>0 ;
$$

et c'est là la formule ci-dessus.

En égalant cette expression à l'intégrale à droite dans (3.), on trouve

$$
\int_{0}^{\infty} \frac{\cos (p \operatorname{arctang} u)}{\left(1+u^{2}\right)^{\frac{1}{2}} p} \cdot \frac{\partial u}{u^{q}}=\frac{\Gamma(p+q-1)}{\Gamma(p) \Gamma(q)} \cdot \frac{\pi}{2 \cos \frac{1}{2} q \pi} ; \quad 1>q>0 .
$$

En substitutant $u=\operatorname{tang} \theta$, on obtient sans difficulté:

4. $\quad \int_{0}^{\frac{1}{2} \pi} \cos ^{p-2} O \cot ^{4} O \cos p O \partial O=\frac{\Gamma(p+q-1)}{\Gamma(p) \Gamma(q)} \cdot \frac{\pi}{2 \cos \frac{1}{2} q \pi} ; \quad 1>q>0$;

ce qui est une formule assez rémarquable.

Pour trouver une autre équation, analogue à la précédente, multiplions (2) par $\frac{\partial u}{u^{4}}$, après y avoir ćcrit $p$ à la place de $\mu$; intégrant alors suivant $u$, on obtient

5. $\int_{0}^{\infty} \frac{\partial u}{u^{4}} \int_{0}^{\infty} x^{p-1} e^{-x} \sin u x \partial x=\Gamma(p) \int_{0}^{\infty} \frac{\sin (p \operatorname{arctang} u)}{\left(1+u^{2}\right)^{\frac{1}{2} p}} \cdot \frac{\partial u}{u^{q}}$, où l'intégrale double à gauche est aussi égale à l'expression

$$
\int_{0}^{\infty} x^{p-1} e^{-x} \partial x \int_{0}^{\infty} \frac{\sin x u}{u^{y}} \partial u,
$$

qui, par la substitution de $u=\frac{t}{u}$ se change en

$$
\left(\int_{0}^{\infty} x^{\nu+q-2} e^{-x} \partial x\right)\left(\int_{0}^{\infty} \frac{\sin t}{t^{q}} \partial t\right)=\Gamma(p+q-1) \cdot \frac{\pi}{2 \Gamma(q) \sin \frac{1}{2}} \bar{q} \pi \quad 2>q>0 .
$$

La valeur de l'intégrale $\int_{0}^{\infty} \frac{\sin t}{t^{q}} \partial t$ peut être trouvée par un calcul tout à fait analogue à celui employé ci-dessus.

En égalant l'expression ci-dessus à l'intégrale à droite dans (5.), on obtient 


$$
\int_{0}^{\infty} \frac{\sin (p \operatorname{arctang} u)}{\left(1+u^{2}\right)^{\frac{1}{2} p}} \cdot \frac{\partial u}{u^{4}}=\frac{\Gamma(p+q-1)}{\Gamma(p) \Gamma(q)} \cdot \frac{\pi}{2 \sin \frac{1}{2} q \pi} ; \quad 2>q>0,
$$

et en fesant $u=\operatorname{tang} \theta$, on en tire la formule

6. $\int_{0}^{\frac{1}{2} \pi} \cos ^{p-2} \theta \cot ^{2} O \sin p O \partial \theta=\frac{\Gamma(p+q-1)}{\Gamma(p) \Gamma(q)} \cdot \frac{\pi}{2 \sin \frac{1}{2} q \pi} ; \quad 2>q>0$.

Les résultats trouvés (4. et 6.) présentent plusieurs formules déjà connues. Si par exemple on fait $q=1$ dans (6.), on parvient à l'équation

$$
\int_{0}^{\frac{1}{2} \pi} \frac{\sin p \theta}{\sin \theta} \cos ^{p-1} O \partial O=\frac{1}{2} \pi
$$

trouvée par Mr. Liouville par une voie tout-différente. (Voyez tome 13. de ce journal page 232.).

II.

Pour faire une autre application de l'équation (1.), multiplions la par

$$
\frac{u^{m} \partial u}{r^{2}+u^{2}}
$$

et prenons en l'intégrale suivant $u$ entre les limites $u=0$ et $u=\infty$. Cela donne

$$
\text { 7. } \int_{0}^{\infty} \frac{u^{m} \cdot \partial u}{r^{2}+u^{2}} \int_{0}^{\infty} x^{\mu-1} e^{-x} \cos u x \partial x=\Gamma(\mu) \int_{0}^{\infty} \frac{\cos (\mu \operatorname{arctang} u)}{\left(1+u^{2}\right)^{\frac{1}{2} \mu}}: \frac{u^{m} \partial u}{r^{2}+u^{2}} \text {. }
$$

Cette intégrale double peut aussi être présentée sous la forme

$$
\int_{0}^{\infty} x^{\mu-1} e^{-x} \partial x \int_{0}^{\infty} \frac{u^{m} \cos x u}{r^{2}+u^{2}} \partial u,
$$

où l'intégration suivant $u$ peut être exécutée au moyen de la formule connue

$$
\int_{0}^{\infty} \frac{u^{m} \cos x u}{r^{2}+u^{2}} \partial u=(-1)^{\frac{1}{2} m} \frac{1}{2} \pi r^{m-1} e^{-r x},
$$

qui suppose que $m$ est un nombre pair. L'intégrale double se change alors en

$$
\int_{0}^{\infty} x^{\mu-1} e^{-x} \partial x(-1)^{\frac{1}{2} m} \frac{1}{2} \pi r^{m-1} e^{-r x}=(-1)^{\frac{1}{2} m} \frac{1}{2} \pi r^{m-1} \frac{\Gamma(u)}{(1+r)^{\prime \prime}},
$$

ce qui est la valeur de l'expression à gauche dans l'équation (7.). On en tire

$$
\text { 8. } \int_{0}^{\infty} \frac{\cos (\mu \operatorname{arctang} u)}{\left(1+u^{2}\right)^{\frac{1}{2} \mu}} \cdot \frac{u^{m} \partial u}{r^{2}+u^{2}}=(-1)^{\frac{1}{2} m} \frac{1}{2} \pi \cdot \frac{r^{m-1}}{(1+r)^{\mu}} \text {. }
$$

Avant de transformer cette intégrale en une autre, qui ne contienne que des fonctions trigonométriques, nous ferons voir qu'on en peut tirer deux autres formules plus générales.

$A$. A cet effet nous présenterons l'équation sous la forme suivante:

$$
\int_{0}^{\infty} \frac{\cos (\mu \operatorname{arctang} u)}{\left(1+u^{2}\right)^{\frac{1}{2} u}} \cdot \frac{u}{r^{2}+u^{2}} u^{m-1} \partial u=(-1)^{\frac{1}{2} m} \frac{1}{2} \pi \cdot r^{m-1}(1+r)^{-\mu} \text {, }
$$




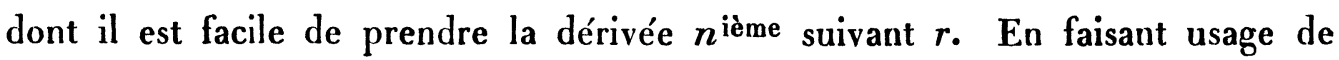
la formule connue

$$
\begin{gathered}
\frac{\partial^{n}[\varphi(r) \psi(r)]}{\partial r^{n}}=\varphi(r) \psi^{(n)}(r)+n_{1} \varphi^{\prime}(r) \psi^{(n-1)}(r)+n_{2} \varphi^{\prime \prime}(r) \psi^{(n-2)}(r)+\ldots \\
{\left[n_{1}=\frac{n}{1}, \quad n^{2}=\frac{n(n-1)}{1.2}, \quad n_{3}=\frac{n(n-1)(n-2)}{1.2 .3}, \text { etc. }\right],}
\end{gathered}
$$

on obtient sans difficulté:

$$
\begin{gathered}
\text { 9. } \int_{0}^{\infty} \frac{\cos (\mu \operatorname{arctang} u)}{\left(1+u^{2}\right)^{\frac{1}{2} \mu}} \cdot \frac{\partial^{n}}{\partial r^{n}}\left[\frac{u}{r^{2}+u^{2}}\right] u^{m-1} \partial u \\
=(-1)^{\frac{1}{2} m+n} \frac{1}{2} \pi(\mu, 1)^{n} \frac{r^{m-1}}{(1+r)^{n+n}}\left\{1-\frac{(m-1) n_{1}}{\mu+n-1}\left(\frac{1+r}{r}\right)+\frac{(m-1)(m-2) n_{2}}{(\mu+n-1)(\mu n-2)}\left(\frac{1+r}{r}\right)^{2}-\ldots .\right\}
\end{gathered}
$$

où $(\mu, 1)^{n}$ designe la factorielle $\mu(\mu+1) \ldots(\mu+n-1)$. être :

Quant à la dérivée sous le signe de l'intégration, elle se trouve

$$
\begin{aligned}
& \frac{\partial^{n}}{\partial r^{n}}\left[\frac{u}{r^{2}+u^{2}}\right]=\frac{1}{2 V-1} \cdot \frac{\partial^{n}}{\partial r^{n}}\left[\frac{1}{r-u V-1}-\frac{1}{r+u V-1}\right] \\
& =\frac{(-1)^{n} 1.2 \ldots n}{2 V-1}\left[\frac{1}{(r-u V-1)^{n+1}}-\frac{1}{(r+u V-1)^{n+1}}\right] \\
& =\frac{(-1)^{n}(1,1)^{n}}{2 V-1} \cdot \frac{(r+u V-1)^{n+1}-(r-u V-1)^{n+1}}{\left(r^{2}+u^{2}\right)^{n+1}} .
\end{aligned}
$$

En posant $r+u V-1=\varrho(\cos \omega+V-1 \sin \omega)$, on aura aussi

$$
\frac{\partial^{n}}{\partial r^{n}}\left[\frac{u}{r^{2}+u^{2}}\right]=(-1)^{n}(1,1)^{n} \frac{\varrho^{n+1}}{\left(r^{2}+u^{2}\right)^{n+1}},
$$

et parcequ'en vertu de l'équation précédente on a $\rho=V\left(r^{2}+u^{2}\right)$ et $\omega=\operatorname{arctang} \frac{u}{r}$ :

$$
\frac{\partial^{n}}{\partial r^{n}}\left[\frac{u}{r^{2}+u^{2}}=(-1)^{n}(1,1)^{n} \frac{\sin \left[(n+1) \operatorname{arctang} \frac{u}{r}\right]}{\left(r^{2}+u^{2}\right)^{\frac{1}{2}(n+1)}}-\right.
$$

L'équation (9.) donne maintenant

$$
\begin{gathered}
\int_{0}^{\infty} \frac{\cos (\mu \operatorname{arctang} u)}{\left(1+u^{2}\right)^{\frac{1}{2} \mu}} \cdot \frac{\sin \left[(n+1) \operatorname{arctang} \frac{u}{r}\right.}{\left(r^{2}+u^{2}\right)^{\frac{1}{2}(n+1)}} u^{m-1} \partial u \\
=(-1)^{\frac{1}{2} m} \frac{1}{2} \pi \cdot \frac{(\mu, 1)^{n}}{(1,1)^{n}} \cdot \frac{r^{m-1}}{(1+r)^{\mu+n}}\left\{1-\frac{(m-1) \cdot n}{1 \cdot(\mu+n-\overline{1})}\left(\frac{1+r}{r}\right)+\frac{(m-1)(m-2) \cdot n(n-1)}{1 \cdot 2 \cdot(\mu+n-1)(\mu+n-2)}\left(\frac{1+r}{r}\right)^{2}-\ldots .\right\}
\end{gathered}
$$

et en fesant $r=1$ et $u=\operatorname{tang} \theta$ : 
18. Schlömilch, développement de quelques intégrales définies.

10. $\int_{0}^{\frac{1}{2} \pi} \cos ^{\mu+n-1} O \operatorname{tang}^{m-1} O \cos \mu \theta \sin (n+1) O \partial \theta$

$=(-1)^{\frac{1}{2} m} \frac{\pi}{2^{\mu+n+1}} \cdot \frac{(\mu, 1)^{n}}{(1,1)^{n}}\left\{1-\frac{(m-1) \cdot n}{1 .(\mu+n-1)} 2+\frac{(m-1)(m-2) \cdot n(n-1)}{1.2 \cdot(\mu+n-1)(\mu+n-2)} 2^{2}-\ldots\right\}$

$B$. L'équation (8.) peut aussi être présentées sous la forme

$$
\int_{0}^{\infty} \frac{\cos (\mu \arctan g u)}{\left(1+u^{2}\right)^{\frac{1}{2}} t^{2}} \cdot \frac{r}{r^{2}+u^{2}} u^{m} \partial u=(-1)^{\frac{1}{2} m} \frac{1}{2} \pi \cdot \frac{r^{m}}{(1+r)^{\prime \prime}} .
$$

En la différentiant $n$ fois de suite, et se reportant à la formule

on en tire

$$
\frac{\partial^{n}}{\partial r^{n}}\left[\frac{r}{r^{2}+u^{2}}\right]=(-1)^{n}(1,1)^{n} \frac{\cos \left[(n+1) \operatorname{arctang} \frac{u}{r}\right]}{\left(r^{2}+u^{2}\right)^{\frac{1}{2}(n+1)}},
$$

$$
\begin{gathered}
\int_{0}^{\infty} \frac{\cos (\mu \operatorname{arctang} u)}{\left(1+u^{2}\right)^{\frac{1}{2}} u} \cdot \frac{\cos \left[(n+1) \operatorname{arctang} \frac{u}{r}\right]}{\left(r^{2}+u^{2}\right)^{\frac{1}{1}(n+1)} u^{m} \partial u} \\
=(-1)^{\frac{1}{2} m} \frac{1}{2} \pi \frac{(\mu, 1)^{n}}{(1,1)^{n}} \cdot \frac{r^{m}}{(1+r)^{\mu+n}}\left\{1-\frac{m \cdot n}{1 \cdot(\mu+n-1)}\left(\frac{1+r}{r}\right)+\frac{m(m-1) \cdot n(n-1)}{1.2 \cdot(\mu+n-1)(\mu+n-2)}\left(\frac{1+r}{r}\right)^{2}-\ldots .\right\}
\end{gathered}
$$

Cela, pour $r=1, u=\operatorname{tang} \theta$, donne

$$
\begin{gathered}
\text { 11. } \int_{0}^{\frac{1}{2} \pi} \cos ^{\mu+n-1} O \operatorname{tang}^{m} O \cos \mu O \cos (n+1) O \circlearrowright O \\
=(-1)^{\frac{1}{2} m} \frac{\pi}{2^{\prime \prime+n}+1} \cdot \frac{(\mu, 1)^{n}}{(1,1)^{n}}\left\{1-\frac{m . n}{1 .(\mu+n-1)} 2+\frac{m(m-1) \cdot n(n-1)}{1.2 \cdot(\mu+n-1)(\mu+n-2)} 2^{2}-\ldots\right\}
\end{gathered}
$$

Voilà une formule correspondante avec celle (10.).

Des calculs tout analogues peuvent être appliqués à la formule (2.). En la multipliant par

$$
\frac{u^{m+1} \partial u}{r^{2}+u^{2}}
$$

et lintégrant suivant $u$, entre les limites $u=0, u=\infty$, on obtient

12. $\int_{0}^{\infty} \frac{u^{m+1} \partial u}{r^{2}+u^{2}} \int_{0}^{\infty} x^{\mu-1} e^{-x} \sin u x \partial x=\Gamma(\mu) \int_{0}^{\infty} \frac{\sin (\mu \operatorname{arctang} u)}{\left(1+u^{2}\right)^{\frac{1}{\mu}} u} \cdot \frac{u^{m+1} \partial u}{r^{2}+u^{2}}$.

Lintégrale double à gauche peut ètre transformée en

$$
\int_{0}^{\infty} x{ }^{n-1} e^{-x} \partial x \int_{0}^{\infty} \frac{u^{m+1} \sin x u}{r^{2}+u^{2}}-\partial u,
$$

où l'intégration peut être operée suivant $u$, si l'on fait usage de la formule

$$
\int_{0}^{\infty} \frac{u^{m+1} \sin x u}{r^{2}+u^{2}}=(-1)^{\frac{1}{2} m} \frac{1}{2} \pi r^{m} e^{-r x}
$$

qui suppose $m$ pair. L'intégrale en question se change maintenant en

$$
\int_{0}^{\infty} x^{\mu-1} e^{-x} \partial x(-1)^{\frac{1}{2} m \frac{1}{2} \pi} r^{m} e^{-r x}=(-1)^{\frac{1}{2} m \frac{1}{2} \pi r^{m}} \frac{\Gamma(\mu)}{(1+r)^{\mu}},
$$

et à l'aide de l'équation (12.) on obtient 
13. $\int_{0}^{\infty} \frac{\sin (\mu \operatorname{arctang} u)}{\left(1+u^{2}\right)^{\frac{1}{2} \mu}} \cdot \frac{u^{m+1} \partial u}{r^{2}+u^{2}}=(-1)^{\frac{1}{2} m} \frac{1}{2} \pi \cdot \frac{r^{m}}{(1+r)^{\mu}}$.

La différentiation $n$ fois répétée de cette équation, donne

$$
\begin{aligned}
& \int_{0}^{\infty} \frac{\sin (\mu \operatorname{arctaug} u)}{\left(1+u^{2}\right)^{\frac{1}{2}} \mu} \cdot \frac{\sin \left[(n+1) \operatorname{arctang} \frac{u}{r}\right]}{\left(r^{2}+u^{2}\right)^{\frac{1}{2}(n+1)}} \frac{-1}{\partial u} \\
& =(-1)^{\frac{1}{2} m} \frac{1}{2} \pi \frac{(\mu, 1)^{n}}{(1,1)^{n}} \cdot \frac{r^{m}}{(1+r)^{\prime \prime+n}}\left\{1-\frac{m \cdot n}{1 .(\mu+n-1)}\left(\frac{1+r}{r}\right)+\frac{m(m-1) \cdot n(n-1)}{1.2 \cdot(\mu+n-1)(\mu+n-2)}\left(\frac{1+r}{r}\right)^{2}-\ldots\right\} \\
& \text { et pour } r=1, u=\operatorname{tang} 0 \text { : }
\end{aligned}
$$

14. $\int_{0}^{\frac{1}{2} \pi} \cos ^{\mu+n-1} O \operatorname{tang}^{m} O \sin \mu O \sin (n+1) O \partial O$

$=(-1)^{\frac{1}{2} m} \frac{\pi}{2^{\mu+n+1}} \cdot \frac{(\mu, 1)^{n}}{(1,1)^{n}}\left\{1-\frac{m \cdot n}{1 .(\mu+n-1)} 2+\frac{m(m-1) \cdot n(n-1)}{1.2 \cdot(\mu+n-1)(\mu+n-2)} 2^{2}-\ldots\right\}$.

Mettant $m-2$ à la place de $m$ et multipliant par $r$, la differentiation donne

$$
\begin{aligned}
& \int_{0}^{\infty} \frac{\sin (\mu \operatorname{arctang} u)}{\left(1+u^{2}\right)^{\frac{1}{2} \mu}} \cdot \frac{\cos \left[(n+1) \operatorname{arctang} \frac{u}{r}\right]}{\left(r^{2}+u^{2}\right)^{\frac{1}{2}(n+1)}} u^{m-1} \cdot \partial u \\
& =(-1)^{\frac{1}{2} m+1} \frac{1}{2} \pi \cdot \frac{(\mu, 1)^{n}}{(1,1)^{n}} \cdot \frac{r^{m-1}}{(1+r)^{\mu+n}}\left\{1-\frac{(m-1) \cdot n}{1 .(\mu+n-1)}\left(\frac{1+r}{r}\right)+\frac{(m-1)(m-2) \cdot n(n-1)}{1.2 \cdot(\mu+n-1)(\mu+n-2)}\left(\frac{1+r}{r}\right)^{2}-\ldots\right\}
\end{aligned}
$$

et pour $r=1, u=\operatorname{tang} 0$ :

15. $\int_{0}^{\frac{1}{2} \pi} \cos ^{\mu+n-1} O \operatorname{tang}^{m-1} O \sin \mu \theta \cos (n+1) O \partial O$

$=(-1)^{\frac{1}{2} m+1} \frac{\pi}{2^{\prime \prime+n+1}} \cdot(1,1)^{n}\left\{1-\frac{(m-1) n}{1 .(\mu+n-1)} 2+\frac{(m-1)(m-2) \cdot n(n-1)}{1.2 \cdot(\mu+n-1)(\mu+n-2)} 2^{2}-\ldots.\right\}$.

Voilà la dernière formule correspondante à celles (10., 11. et 14.). Comme toutes ces intégrales contiennent trois nombres $\mu, m, n$, dont le premier est absolument arbitraire, on en peut tirer beaucoup de formules particulières plus où moins connues.

III.

Passons maintenant à une troisième application des équations (1.) et (2). Multipliant par

$$
\frac{\partial u}{\left(r^{2}+u^{2}\right)^{n+1}}
$$

et intégrant suivant $u$, on tire de (1):

16. $\int_{0}^{\infty} \frac{\partial u}{\left(r^{2}+a^{2}\right)^{n+1}} \int_{0}^{\infty} x^{u-1} e^{-x} \cos u x \partial x=\Gamma(\mu) \int_{0}^{\infty} \frac{\cos (\mu \operatorname{arctang} u)}{\left(1+u^{2}\right)^{\frac{1}{2} \mu}} \cdot \frac{\partial u}{\left(r^{2}+u^{2}\right)^{n+1}}$.

Renversant l'ordre des intégration, lintćgrale à gauche se change en 
18. Schlömilch, développement de quelques intégrales définies.

$$
\int_{0}^{\infty} x^{u-1} e^{-x} \partial x \int_{0}^{\infty} \frac{\cos x u \partial u}{\left(r^{2}+u^{2}\right)^{n+1}}
$$

où lintégration suivant $u$ peut être effectuée par le secours de la formule connue

$$
\begin{gathered}
\int_{0}^{\infty} \frac{\cos x u \partial u}{\left(r^{2}+u^{2}\right)^{n+1}} \\
=\frac{\pi e^{-r_{x}}}{2^{n+1} \Gamma(n+1)}\left\{M_{0} \frac{x^{n}}{r^{n+1}}+M_{2} \frac{x^{n-1}}{r^{n+2}}+M_{4} \frac{x^{n-2}}{r^{u+3}}+\ldots\right\},
\end{gathered}
$$

dans laquelle les coefficients $M_{0}, M_{2}, M_{4}$ etc. sont déterminés par les é(juations

17. $M_{0}=1, \quad M_{2}=\frac{1}{2}(n+1) n, \quad M_{4}=\frac{(n+2)(n+1) n(n-1)}{2.4}$,

$$
M_{6}=\frac{(n+3)(n+2)(n+1) n(n-1)) n-2)}{2.4 .6}, \quad \text { etc. }
$$

Maintenant on obtient

$$
\begin{aligned}
& \int_{0}^{\infty} x^{u-1} e^{-x} \partial x \int_{0}^{\infty} \frac{\cos x u \partial u}{\left(r^{2}+u^{2}\right)^{n+1}} \\
& =\frac{\pi}{2^{n+1} \Gamma(n+\overline{1})}\left\{\frac{M_{0}}{r^{n+1}} \int_{0}^{\infty} x^{\mu+n-1} e^{-(1+r) x} \partial x+\frac{M_{2}}{r^{n+2}} \int_{0}^{\infty} x^{\mu+n-2} e^{-(1+r) x} \partial x\right. \\
& \left.+\frac{M_{4}}{r^{n+3}} \int_{0}^{\infty} x^{\mu+n-3} e^{-(1+r) x} \partial x+\ldots\right\}
\end{aligned}
$$

et en opérant les intégrations indiquées à droite, on trouve que l'intégrale double mentionnée est égale à la série finie

$$
\frac{\pi}{2^{n+1} \Gamma(n+1)}\left\{\frac{M_{0}}{r^{n+1}} \cdot \frac{\Gamma(\mu+n)}{(1+r)^{\mu}+n}+\frac{M_{2}}{r^{n+2}} \cdot \frac{\Gamma(\mu+n-1)}{(1+r)^{\mu}+n-1}+\frac{M_{4}}{r^{n-3}} \cdot \frac{\Gamma(\mu+n-2)}{(1+r)^{\prime \prime+n-2}}+\ldots\right\},
$$

ou bien à

$$
\begin{gathered}
\frac{\pi}{\Gamma(n+1)(2 r)^{n+1}(1+r)^{\mu+n}}\left\{M_{0} \Gamma(\mu+n)+M_{2} \Gamma(\mu+n-1) \frac{1+r}{r}\right. \\
\left.+M_{4} \Gamma(\mu+n-2)\left(\frac{1+r}{r}\right)^{2}+\ldots\right\}
\end{gathered}
$$

En observant qu'en vertu des propriétés des intégrales Euleriennes on a

$$
\Gamma(\mu+n-1)=\frac{\Gamma(\mu+n)}{\mu+n-1} \quad \text { et } \quad \Gamma(\mu+n-2)=\frac{\Gamma(\mu+n)}{(\mu+n-1)(\mu+n-2)} \quad \text { etc. }
$$

la série précédente peut aussi être présentée sous la forme

$$
\frac{\pi \Gamma(\mu+r)}{\Gamma(n+1)(2 r)^{n+1}(1+r)^{\mu+n}}\left\{M_{0}+\frac{M_{2}\left(1+\frac{1}{r}\right)}{\mu+n-1}+\frac{M_{4}\left(1+\frac{1}{r}\right)}{(\mu+n-1)(\mu+n-2)}+\ldots\right\} .
$$

Cela étant la valeur de l'intégrale double en (16.), nous avons maintenant: 


$$
\begin{gathered}
\int_{0}^{\infty} \frac{\cos (\mu \operatorname{arctang} u)}{\left(1+u^{2}\right)^{\frac{1}{2} \mu}} \cdot \frac{\partial u}{\left(r^{2}+u^{2}\right)^{n+1}} \\
\frac{\pi \Gamma(\mu+n): \Gamma(\mu)}{\Gamma(n+1)(2 r)^{n+1}(1+r)^{u}+n}\left\{M_{0}+\frac{M_{2}\left(1+\frac{1}{r}\right)}{\mu+n-1}+\frac{M_{4}\left(1+\frac{1}{r}\right)^{2}}{(\mu+n-1)(\mu+n-2)}+\ldots\right\},
\end{gathered}
$$

où $\frac{\Gamma(\mu+n): \Gamma(\mu)}{\Gamma(n+1)}$ peut être remplacé par

$$
\frac{\mu(\mu+1)(\mu+2) \ldots(\mu+n-1)}{1.2 .3 \ldots . . n}=\frac{(\mu, 1)^{n}}{(1,1)^{n}} .
$$

Fesant enfin $r=1, u=\operatorname{tang} \theta$, nous aurons en vertu des valeurs de $M_{0}$, $M_{2}, M_{4}$ etc.

$$
\begin{gathered}
\text { 18. } \quad \int_{0}^{\frac{l}{2} \pi} \cos ^{\mu+2 n} O \cos \mu O \partial \theta \\
=\frac{\pi}{2^{\mu+2 n+1}} \cdot \frac{(\mu, 1)^{n}}{(1,1)^{n}}\left\{1+\frac{(n+1) n}{1 .(\mu+n-1)}+\frac{(n+2)(n+1) n(n-1)}{1.2 \cdot(\mu+n-1)(\mu+n-2)}+\ldots\right\} .
\end{gathered}
$$

Un calcul semblable donnera un théorème analogue; car de (2.)

on tire

19. $\int_{0}^{\infty} \frac{u \partial u}{\left(r^{2}+u^{2}\right)^{n+1}} \int_{0} x^{\mu-1} e^{-x} \sin u x \partial x=\Gamma(\mu) \int_{0}^{\infty} \frac{\sin (\mu \operatorname{arctang} u)}{\left(1+u^{2}\right)^{\frac{1}{2}, u}} \cdot \frac{u \partial u}{\left(r^{2}+u^{2}\right)^{n+1}}$, où lintégrale double peut être transformée en

$$
\int_{0}^{\infty} x^{\mu-1} e^{-x} \partial x \int_{0}^{\circ} \frac{u \sin x u}{\left(r^{2}+u^{2}\right)^{n+1}}
$$

La valeur de cette intégrale par rapport à $u$ est, comme on sait,

$$
\frac{\pi e^{-r x}}{2^{n+1} \Gamma(n+\overline{1})}\left\{N_{0} \frac{x^{n}}{r^{n}}+N_{2} \frac{x^{n-1}}{r^{n+1}}+N_{4} \frac{x^{n-2}}{r^{n+2}}+\ldots\right\}
$$

où

$$
\begin{gathered}
N_{0}=1, \quad N_{2}=\frac{1}{2}(n-1), \quad N_{4}=\frac{(n+1) n(n-1)(n-2)}{2.4}, \\
N_{6}=\frac{(n+2)(n+1) n(n-1)(n-2)(n-3)}{2.4 .6}, \quad \text { etc. }
\end{gathered}
$$

Delà on tire sans difficulté la valeur suivante de lintégrale double:

$$
\frac{\pi}{2^{n+1} \Gamma(n+1)}\left\{\frac{N_{0}}{r^{n}} \cdot \frac{\Gamma(\mu+n)}{(1+r)^{\prime \prime+n}}+\frac{N_{2}}{r^{n+1}} \cdot \frac{\Gamma(\mu+n-1)}{(1+r)^{\prime \prime+n-1}}+\frac{N_{4}}{r^{n+2}} \cdot \frac{\Gamma(\mu+n-2)}{(1+r)^{\prime \prime+n-2}}+\ldots\right\},
$$
ou bien

$$
\frac{r \pi \Gamma(\mu+n)}{\Gamma(n+1)(2 r)^{n+1}(1+r)^{\mu+n}}\left\{N_{0}+\frac{N_{2}\left(1+\frac{1}{r}\right)}{\mu+n-1}+\frac{N_{4}\left(1+\frac{1}{r}\right)^{2}}{(\mu+n-1)(\mu+n-2)}+\ldots\right\},
$$

et en égalant cette valeur à l'intégrale à droite dans (19.): 


$$
\begin{gathered}
\int_{0}^{\infty} \frac{\sin (\mu \operatorname{arctang} u)}{\left(1+u^{2}\right)^{\frac{1}{2} \mu}} \cdot \frac{u \partial u}{\left(r^{2}+u^{2}\right)^{n+1}} \\
=\frac{r \pi \Gamma(\mu+n): \Gamma(\mu)}{\Gamma(n+1)(2 r)^{n+1}(1+r)^{\mu+n}}\left\{N_{0}+\frac{N_{2}\left(1+\frac{1}{r}\right)}{\mu+n-1}+\frac{N_{4}\left(1+\frac{1}{r}\right)}{(\mu+n-1)(\mu+n-2)}+\ldots\right\}
\end{gathered}
$$

Fesant enfin $r=1, u=\operatorname{tang} \theta$, on obtient

$$
\begin{gathered}
\text { 20. } \int_{0}^{\frac{1}{2} \mu} \cos ^{\mu+2 n} \theta \sin \mu \theta \operatorname{tang} \theta \partial \dot{ } \\
=\frac{\pi}{2^{\mu+2 n+1}} \cdot \frac{(\mu, 1)^{n}}{(1,1)^{n}}\left\{1+\frac{n(n-1)}{1 .(\mu+n-1)}+\frac{(n-1) n(n-1)(n-2)}{1.2 \cdot(\mu+n-1)(\mu+n-2)}+\ldots .\right\}
\end{gathered}
$$

Il est à remarquer quill n'est pas permis de supposer $\mu=0$, ou négatif, dans toutes les formules que nous venons de présenter, parceque le calcul a été fondé sur les propriétés des intégrales Eulériennes qui n'ont lieu que pour des valeurs positives de la variable. 